\title{
New Tools for Complex Assessment of Patients after Myocardial Infarction
}

\author{
Piotr Michalski ${ }^{1 *}$ Agata Kosobucka $^{1 *}{ }^{*}$ Jakub Ratajczak $^{1,2}$, Michal Siedlaczek $^{1,2}$ and Lukasz Pietrzykowski ${ }^{1}$ \\ ${ }^{1}$ Department of Health Promotion, Collegium Medicum in Bydgoszcz, Poland \\ ${ }^{2}$ Department of Cardiology and Internal Medicine, Col-legium Medicum in Bydgoszcz, Poland \\ *Corresponding author: Piotr Michalski, Department of Health Promotion, Nicolaus Copernicus University, Collegium \\ Medicum, ul. Lukasiewicza St.1, 85-821 Bydgoszcz, Poland
}

Agata Kosobucka, Department of Health Promotion, Nicolaus Copernicus University, Collegium Medicum, ul. Lukasiewicza St.1,

85-821 Bydgoszcz, Poland

\section{ARTICLE INFO}

Received: 幽 April 04, 2019

Published: 慧 April 10, 2019

Citation: Piotr M Agata K, Jakub R, Michal S, Lukasz P. New Tools for Complex Assessment of Patients after Myocardial Infarction. Biomed J Sci \& Tech Res 16(5)-2019. BJSTR. MS.ID.002928.

Keywords: Chronic Disease; Myocardial Infarction; Adherence; Non-Adherence; Readiness from Hospital Discharge

\section{ABSTRACT}

Cardiovascular diseases are serious health, social, and economical problem mainly due to high morbidity and mortality. Myocardial infarction is burdened with one of the highest mortality rates and therefore requires particular interest of the researchers. The high mortality during long-term follow-up could be potentially explained by patients' attitude and not following the therapeutic recommendation which is defined in the literature as non-adherence. It is estimated that in patients with low adherence the risk of death is two times higher than in those who followed the therapeutic instructions. In order to improve the long-time results, we developed free, publicly available tools for complex assessment of patients after myocardial infarction. Those scales were designed to assess patient's: educational needs and readiness for hospital discharge, functioning in the chronic illness, and adherence to recommendations regarding pharmacotherapy.

Abbreviations: RHD-MIS: The Readiness from Hospital Discharge of patients after Myocardial Infarction Scale; FCIS: The Functioning in the Chronic Illness Scale; ACDS: The Adherence in Chronic Disease Scale

\section{Introduction}

Cardiovascular diseases are serious health, social, and economical problem mainly due to high morbidity and mortality. Myocardial infarction is burdened with one of the highest mortality rates and therefore requires particular interest of the researchers $[1,2]$. Coordinated and complex treatment strategies with modern diagnostic and therapeutic techniques definitely contributed to improving the survival of patients after myocardial infarction. However, development in the field of interventional cardiology and cardiac surgery as well as pharmacotherapy might still be insufficient to significantly improve the health of the society [3]. In-hospital mortality rate reported in previous studies of patients after myocardial infarction was $6 \%$ and $4.9 \%$ for those with and without ST segment elevation respectively. Long-term observation provided more alarming results since up to $20 \%$ of patients died within the first year after the myocardial infarction [4].
The high mortality during long-term follow-up could be potentially explained by patients' attitude and not following the therapeutic recommendation which is defined in the literature as non-adherence. According to World Health Organization (WHO), only $50 \%$ of patients with chronic diseases in highly developed countries implement the medical recommendations in the daily routine [5]. It is estimated that in patients with low adherence the risk of death is two times higher than in those who followed the therapeutic instructions [6]. In order to improve the long-time results, we developed free, publicly available tools for complex assessment of patients after myocardial infarction. Those scales were designed to assess patient's: educational needs and readiness for hospital discharge, functioning in the chronic illness, and adherence to recommendations regarding pharmacotherapy (available on the website of the Department of Health Promotion, Collegium Medicum, Nicolaus Copernicus University, Poland 
https://www.wnoz.cm.umk.pl/kizprzdr/narzedzia-badawczeresearch-tools/).

\section{Tools Description}

The Readiness from Hospital Discharge of patients after Myocardial Infarction Scale (RHD-MIS) is a validated tool ( $\alpha$-Cronbach 0.789 ) available in two versions, first for the patients and the second for the researcher. The questionnaire consists of 27 questions divided into 4 parts: 3 scoring and 1 non-scoring subscale. Two of the scoring sub-scales are prepared for subjective and objective assessment of patient's knowledge and the third is designed to determine the patient's expectations. The last nonscoring sub-scale regards the opinion about the disease. Patient can achieve low, medium, or high score in each sub-scale as well as in total for the entire scale [7]. The Functioning in the Chronic Illness Scale (FCIS) is a new diagnostic tool validated ( $\alpha$-Cronbach 0.855 ) on the group of 366 patients with acute coronary syndromes. The patient's functioning is examined in 3 aspects: the impact of illness on the patients, the patient's impact on the illness, and the impact of illness on the patient's attitude.

The patient provides one answer scored on a scale from 1 to 5 points for each of 24 questions included in the questionnaire. In each investigated aspect of functioning as well as for the total score 3 intensity levels were distinguished: low, medium, and high. High scores achieved in particular sub-scales suggests no impact of the disease on the patients, adequate patient's opinion in the illness, and active attitude in coping with the disease. Higher overall score reflects very good functioning in the chronic illness [8]. The Adherence in Chronic Disease Scale (ACDS) is used for assessment of adherence level regarding pharmacotherapy in patients with chronic diseases. ACDS is a free, publicly available tool validated ( $\alpha$-Cronbach 0.739 ) on the group of 300 patients suffering from cardiovascular diseases. The scale consists of 7 questions with proposed answered scored from 0 to 4 points. First 5 questions address behaviors directly determining adherence and last two regards situations and opinions indirectly influencing the therapeutic process [9].

\section{Reasoning}

Socioeconomic, condition-related, healthcare systemrelated, treatment option-related and other patient-dependent factors were distinguished by WHO as 5 major determinants of non-adherence $[3,10]$. Presented division shows how complex and difficult could be the achievement of optimal adherence. It requires multidirectional actions focused not only on patient's somatic state but also concentrated on mental and social aspects. It should be underlined that during acute phase of the disease the healthcare providers supervise the proper course of the treatment. However, after hospital discharge the realization of therapeutic recommendations depends mainly on the patient. The assessment of the patient's readiness to hospital discharge is recommended since it can be helpful to identify those patients who could require some additional actions of healthcare team. Widely understood, effective education reduces the rates of complications, rehospitalizations, and significantly improves patient comfort and sense of security and by that also the adherence [11-13].
The RHD-MIS scale deserves special attention, because it gives the opportunity to compare the subjective and objective knowledge of the patient and to understand the expectations of further education. Applying it in practice makes it possible to prepare a long-term patient education plan. The significance of health education in the rehabilitation process is underlined in the Canadian Assistance of Cardiac Rehabilitation Guidelines, which indicates that it should be individualized and carried out by a team of professionals. Additional task of health education is to support, motivate to change behaviors and persevere in the actions taken $[14,15]$. Patients included in educational activities show a higher level of knowledge and are often characterized by appropriate health behaviors in terms of diet, exercise, and smoking cessation. Unfortunately, as indicated by many scientific reports, both the health behaviors [16,17] and adherence [18,19] remain unsatisfactory in the population of patients with cardiovascular diseases. In the literature, there are many reports evaluating the functioning of patients with respect to mental and physical sphere.

However, it should be noted that the patient's attitude to the disease implies his reactions which directly affect the therapeutic process. Up to now, no studies have been published to assess so defined functioning in chronic disease, apart from the FCIS validation study [8]. Facing the illness patients activate mechanisms aimed at dealing with the problem. Active behaviors, focusing on searching for information to control and influence their own disease, seem to be the most beneficial. However, subjective, often inadequate to the current state, perception of the disease could lead to the attitude unfavourable for treatment: denial, anxiety or avoidance [20]. Insufficient discipline of patients in the implementation of the therapeutic plan and the lack of cooperation of medical personnel with the patient contribute to the incorrect control of risk factors, which in turn leads to disease progression and rehospitalizations. The ACDS questionnaire, was prepared upon our own observations and experiences and can be helpful to diagnose the most common incorrect behaviors of patients and the specific attitudes of medical personnel.

\section{Conclusion}

The occurrence of various complications and even premature death may be caused by the dose omission, self-correction of the dose and early termination of pharmacotherapy [21,22]. Particularly vulnerable groups are the elderly, lonely, low educated and with poor economic status, as well as patients with comorbidities and depression [23-25]. The multidimensionality of the problem of non-compliance with the therapeutic plan is a real challenge for health care workers and the healthcare system. The presented tools are designed to improve the recognition of potential causes of ineffective education and in longer perspective, failure to comply with the recommendations.

\section{Acknowledgement}

The authors wish to thank Prof. Aldona Kubica (Nicolaus Copernicus University, Collegium Medicum, Poland) for valuable discussion of this work and for careful support. 


\section{References}

1. Nichols M, Townsend N, Scarborough P, Rayner M (2014) Cardiovascular disease in Europe 2014: epidemiological update. Eur Heart J 35(42): 2950-2959.

2. World Health Organization. Mortality and global burden of disease.

3. Kubica A, Obońska K, Kasprzak M, Sztuba B, Navarese EP, et al. (2016) Prediction of high risk of non-adherence to antiplatelet treatment. Kardiol Pol 74(1): 61-67.

4. Garcia - Garcia C, Subirana I, Sala J, Bruguera J, Sanz G, et al. (2011) Long-term prognosis of first myocardial infarction according to the electrocardiographic pattern (ST-elevation myocardial infarction, nonST-elevation myocardial infarction and non-classified myocardial infa) and revascularization procedures. Am J Cardiol 108(8): 1061-1067.

5. Sabaté E (2003) Adherence to long-term therapies: evidence for action. World Health Organization, Geneva.

6. Simpson SH, Eurich DT, Majumdar SR, Padwal RS, Tsuyuki RT, et al. (2006) A meta-analysis of the association between adherence to drug therapy and mortality. BMJ 33(7557): 1-6.

7. Buszko K, Kosobucka A, Michalski P, Pietrzykowsk Ł, Jurek A, et al. (2017) The readiness from hospital discharge of parients after acute myocardial infarction: a new self-reported questionnaire. Med Res J 2(1): 20-28.

8. Buszko K, Pietrzykowski Ł, Michalski P, Kosobucka A, Stolarek W, et al. (2018) Validation of the Functioning in Chronic Ilness Scale. Med Res J 3(2): 63-69.

9. Kubica A, Kosobucka A, Michalski P, Pietrzykowski Ł, Jurek A, et al (2017) The Adherence in Chronic Diseases Scale - a new tool to monitor implementation of a treatment plan. Folia Cardiologica 12,7: 19-26.

10. Kardas P, Lewek P, Matyjaszczyk M (2013) Determinants of patients adherence: a review of systematic reviews. Front Pharmacol 4: 91.

11. Kubica A, Kosobucka A, Fabiszak T, Gorog DA, Siller-Matula JM (2019) Assessment of adherence to medication in patients after myocardial infarction treated with percutaneous coronary intervention. Is there a place for new self-reported questionnaires? Curr Med Res Opin 35(2): 341-349.

12. Weiss ME, Costa LL, Yakusheva O, Bobay KL (2014) Validation of patient and nurse short forms of the readiness for hospital discharge scale and their relationship to return to the hospital. Health Services Research 49(1): 304-317.

13. Weiss ME, Piacentine LB, Lokken L, Ancona J, Archer J, et al. (2007) Perceived readiness for hospital discharge in adult medical- surgical patients. Clin Nurse Spec 21(1): 31-42.

\section{ISSN: 2574-1241}

DOI: 10.26717/BJSTR.2019.16.002928

Piotr Michalski, Agata Kosobucka. Biomed J Sci \& Tech Res

This work is licensed under Creative Commons Attribution 4.0 License

Submission Link: https://biomedres.us/submit-manuscript.php
14. Stone AJ, Arthur AM, Suskin M (2009) Canadian guidelines for cardiac rehabilitation and cardiovascular disease prevention: translating knowledge to action. Canadian Association of Cardiac Rehabilitation and Cardiovascular Prevention. $3^{\text {rd }}$ (edn.).

15. Ghist GLM, Abdallah F, Grace SL, Thomas S, Oh P (2014) A systematic review of patients education in cardiac patients: Do they increase knowledge and promote health behavior change? Patient Educ Couns 95(2): 160-174.

16. Kotseva K, De Backer G, De Bacquer D, Lars Ryden, Arno Hoes, et al. (2019) Lifestyle and impact on cardiovascular risk factor in coronary patients across 27 countries: Result from the European Society of Cardiology ESC-EROP EuropAspire V registry. Eur J Prev Cardiol.

17. Kotseva K, De Bacqer D, Jennings C (2016) Adverse lifestyle trends counter improvements in cardiovascular risk factors and therapeutic managements of coronary patients from twenty-four European countries. Eur J Prev Cardiol 23: 636-648.

18. Kosobucka A, Michalski P, Pietrzykowski Ł, Kasprzak M, Obońska K, et al. (2018) Adherence to treatment assessed with the Adherence in Chronic Diseases Scale in patients after myocardial infarction. Patient Preference and Adherence 12: 333-340.

19. Kubica A, Kasprzak M, Obońska K, Fabiszak T, Laskowska E, et al. (2015) Discrepancies in assessment of adherence to antiplatelet treatment after myocardial infarction. Pharmacology 95(1-2): 50-58.

20. Leksowska A, Jaworska I, Gorczyca P (2011) Choroba somatyczna jako wyzwanie adaptacyjne dla człowieka. Folia Cardiologica Excerpta 6(4): 244-248.

21. Fitzgerald AA, Powers JD, Ho PM, Maddox PM, Peterson PN, et al. (2011) Impact of medication non-adherence on hospitalizations and mortality in heart failure. J Card Fail 17(8): 664-669.

22. Desai NR, Choudry NK (2013) Impediments to adherence to post myocardial infarction medications. Curr Cardiol Rep 15(1): 322.

23. Czarny MJ, Nathan AS, Yeh RW, Mauri L (2014) Adherence to dual antiplatelet therapy after coronary stenting: A systematic review. Clinic Cardiol 37(8): 505-513.

24. Nordstrom BL, Simeone JC, Zhao Z, Molife C, McCollam PL, et al. (2013) Adherence and persistence with prasugrel following acute coronary syndrome with percutaneous coronary intervention. Am J Cardiovasc Drugs 13(4): 263-271.

25. Quadros AS, Welter DI, Camozzatto FO, Chaves A, Mehta RH, et al. (2011) Identifying patients at risk for premature discontinuation of thienopyridine after coronary stent implantation. Am J Cardiol 107(5): 685-689.

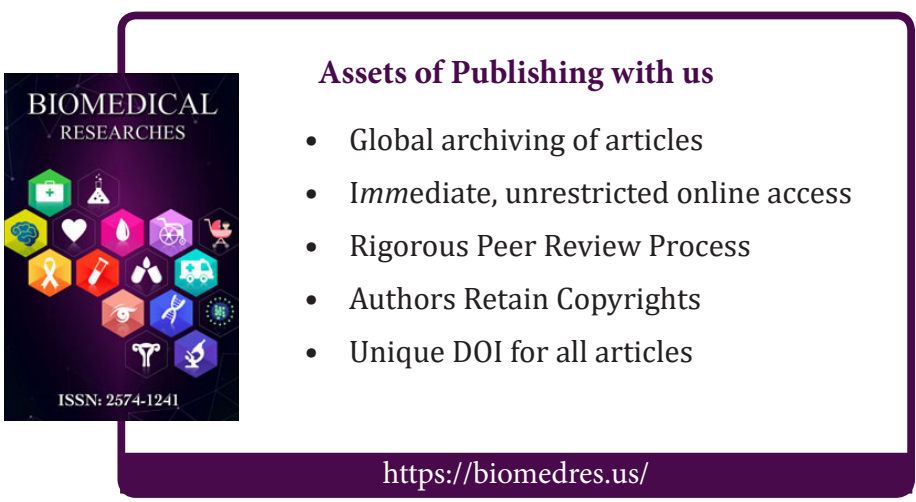

Copyright@ Piotr Michalski, Agata Kosobucka | Biomed J Sci \& Tech Res| BJSTR. MS.ID.002928. 